\title{
Surveillance of hospitalizations with pandemic A(H1N1) 2009 influenza infection in Queensland, Australia
}

\author{
Hai Phung, ${ }^{a, b}$ Frank Beard, ${ }^{a}$ Christine Selvey, ${ }^{a}$ Ranil Appuhamy ${ }^{a}$ and Frances Birrellla,b \\ Correspondence to Hai Phung (e-mail: Hai_Phung@health.qld.gov.au).
}

Objective: To describe the demographic and clinical characteristics of patients hospitalized with pandemic A(H1N1) 2009 infection in Queensland, Australia between 25 May and 3 October 2009 and to examine the relationship between timing of antiviral treatment and severity of illness.

Method: Using data from the Queensland Health EpiLog information system, descriptive analysis and logistic regression modelling were used to describe and model factors which influence patient outcomes (death, admission to intensive care unit and/or special care unit). Data on patients admitted to hospital in Queensland with confirmed pandemic $\mathrm{A}(\mathrm{H} 1 \mathrm{~N} 1) 2009$ infection were included in this analysis.

Results: 1236 patients with pandemic $A(\mathrm{H} 1 \mathrm{~N} 1) 2009$ infection were admitted to hospitals in Queensland during the study period. Of the total group: $15 \%$ were admitted to an intensive care unit or special care unit; $3 \%$ died; $34 \%$ were under the age of 18 years and $8 \%$ were 65 years of age or older; and $55 \%$ had at least one underlying medical condition. Among the 842 patients for whom data were available regarding the use of antiviral drugs, antiviral treatment was initiated in 737 (87.5\%) patients, treatment commenced at a median of one day (range 1-33 days) after onset of illness. Admission to an intensive care unit or special care unit (ICU/SCU) or death was significantly associated with increased age, lack of timeliness of antiviral treatment, chronic renal disease and morbid obesity.

Discussion: Early antiviral treatment was significantly associated with lower likelihood of ICU/SCU admission or death. Early antiviral treatment for influenza cases may therefore have important public health implications.

$\mathrm{T}$ he first case of pandemic $A(H 1 N 1) 2009$ influenza infection in Australia was reported in a 28-year-old female in Queensland on 9 May 2009. Queensland, along with other Australian states and territories, invested in intense public health efforts to manage and control the outbreak. ${ }^{1}$ Information on the clinical spectrum of pandemic $A(H 1 N 1) 2009$ illness and factors associated with admission to hospital is scarce in Australia. Internationally, it had been reported that the majority of the early cases reported mild, influenzalike illnesses with fever and respiratory symptoms, but more severe infections have also occurred..$^{2-6}$ Obesity, underlying health conditions and delayed neuraminidase inhibitor treatment were the major risk factors for a poor outcome of infection. ${ }^{4,5}$ Risk factors for poor clinical outcomes for pandemic A(H1N1) 2009 infection in Australia warrant further investigation to help clinicians indentify patients at high risk of severe disease.

Several surveillance systems were used to monitor and evaluate the progression of the outbreak in Queensland. These systems initially focused on notification data from public health units and laboratories. However, as the pandemic progressed, hospitalization data became of paramount importance for health planners. A new state-wide hospital-based surveillance application, EpiLog, was developed to monitor real-time admissions of patients with pandemic A(H1N1) 2009 infection to all public hospitals in Queensland. Data were also received from major private hospitals. This allowed monitoring of the outbreak and its impact on the hospital system.

We present findings from a retrospective analysis of data collected by this new surveillance system. The aims of the analysis were to describe the demographic and clinical characteristics of patients hospitalized with pandemic $A(H 1 N 1) 2009$ infection in Queensland (between 25 May and 3 October 2009) and to examine the relationship between timing of antiviral treatment and severity of illness.

\footnotetext{
a Communicable Diseases Branch, Queensland Health, Brisbane, Queensland, Australia.

b School of Public Health, Queensland University of Technology, Brisbane, Queensland, Australia.

Submission date: 29 November 2010; Publication date: 30 May 2011

doi: 10.5365/wpsar.2010.1.1.013
} 


\section{METHODS AND DATA COLLECTION}

The data for this analysis were extracted from EpiLog. This web-based application was developed in Queensland for the surveillance of patients admitted to public hospitals with suspected or confirmed pandemic A(H1N1) 2009 influenza infection. The same data elements were provided by major private hospitals using a purposedesigned Microsoft Excel spreadsheet.

Data elements entered into EpiLog included pandemic $A(H 1 N 1) 2009$ influenza infection status, self-reported medical conditions (morbid obesity, pregnancy, immunocompromised status, diabetes, chronic respiratory disease, renal disease, and cardiac disease), time of antiviral treatment initiation and intubation or ventilation status. EpiLog is linked to the Hospital Based Clinical Information System (HBCIS) used in public hospitals, which stores data relating to patient demographics and hospital stay with a unique patient identifier. Patient demographic data, admission and discharge details and ward transfer details were updated in EpiLog via this interface.

Data were extracted from EpiLog for analysis and aggregated with data received from private hospitals. Data on patients with confirmed pandemic A(H1N1) 2009 influenza infection who were admitted to hospitals in Queensland between 25 May and 3 October 2009 were analysed. Admission to an intensive care unit (ICU) or a special care unit (SCU) was used as a proxy for severity of illness.

\section{Statistical analysis}

The proportion of ICU/SCU admissions among hospitalized patients with pandemic $A(H 1 N 1) 2009$ infection was calculated. Univariate association of various factors were evaluated using descriptive statistical techniques. The association between ICU/SCU admission and patient characteristics (age, sex, indigenous status, underlying medical conditions and whether antiviral treatment was received) was evaluated using univariate logistic regression followed by multivariate forward stepwise logistic regression test.

To assess the predictive value of various factors, the area under the receiver-operating characteristic (ROC) curves was calculated. The ROC curve evaluates the ability of the full logistic regression model to discriminate ${ }^{7}$ patients admitted to ICU/SCU from those with no ICU/
SCU admission. The SPSS package (Version 12) was used for all analyses. ${ }^{8}$

Ethical clearance was not required for this analysis. This analysis was conducted as a clinical audit (during a public health emergency event), required under the Health Services Act (1991).

\section{RESULTS}

\section{Patient characteristics}

There were 1236 hospitalized patients recorded in Queensland with confirmed pandemic A(H1N1) 2009 infection between 25 May and 3 October 2009. Table 1 summarizes selected demographic and clinical characteristics of these patients. The median age was 29 years (range, less than 1 month to 90 years). Eighty-four patients $(7 \%)$ were less than one year of age, 420 (34\%) were under 18 years of age and $96(8 \%)$ were aged 65 years or over. Of the total study sample, $686(55 \%)$ were recorded as having at least one underlying medical condition and $162(13 \%)$ had at least two such conditions. A total of 80 patients were pregnant $(31 \%$ of female patients between $18-45$ years old). Sixty patients (5\%) were morbidly obese, with all but one of these patients (98\%) being aged 25 years or older. Fifteen per cent of the patients admitted to hospital with confirmed pandemic $A(\mathrm{H} 1 \mathrm{~N} 1)$ 2009 infection were indigenous.

Antiviral drug administration data were available for 842 patients, with 703 (83\%) recorded as receiving oseltamivir, $9(1 \%)$ zanamivir and $25(3 \%)$ treated with an unspecified antiviral and $105(13 \%)$ as not having received any antiviral drugs. The median time from the onset of illness to the initiation of antiviral therapy was one day (range, less than 1 day to 33 days). Among patients for whom data on antiviral therapy timing were available (842), 561 (67\%) received antiviral treatment within 48 hours of the onset of symptoms.

\section{Intensive care unit and special care unit admissions and mortality}

Patients admitted to ICU/SCU represented 189 of the 1236 (15\%) patients. The median age was 49 years (range, 1 to 84 years). Patients who were admitted to ICU or SCU were more likely to be older, have diabetes or chronic cardiac or renal disease, be morbidly obese and not have received antiviral treatment within 48 hours of 
Table 1. Characteristics of hospitalized patients with confirmed pandemic A(H1N1) 2009 infection ( $\mathrm{N}=1236)$, Queensland, Australia (May to October 2009)

\begin{tabular}{|c|c|c|}
\hline & Frequency & $(\%)$ \\
\hline \multicolumn{3}{|l|}{ Hospital Type } \\
\hline Public & 1019 & $(17.5)$ \\
\hline Private & 217 & $(82.5)$ \\
\hline \multicolumn{3}{|l|}{ Patient's age (years) } \\
\hline Less than 10 & 305 & $(24.7)$ \\
\hline $10-17$ & 115 & (9.3) \\
\hline $18-24$ & 109 & (8.8) \\
\hline $25-44$ & 280 & $(22.7)$ \\
\hline $45-64$ & 326 & $(26.4)$ \\
\hline $65+$ & 96 & (7.8) \\
\hline \multicolumn{3}{|l|}{ Indigenous status } \\
\hline Indigenous & 191 & $(15.4)$ \\
\hline Non-indigenous & 924 & $(74.8)$ \\
\hline Unknown & 121 & $(9.7)$ \\
\hline \multicolumn{3}{|l|}{ Sex } \\
\hline Male & 568 & $(45.9)$ \\
\hline Female & 669 & $(54.1)$ \\
\hline \multicolumn{3}{|l|}{ Underlying medical conditions } \\
\hline Chronic respiratory disease & 415 & (33.5) \\
\hline Diabetes & 122 & (9.9) \\
\hline Morbid obesity & 60 & $(4.9)$ \\
\hline Immunocompromised & 87 & (7.0) \\
\hline Chronic cardiac disease & 97 & (7.6) \\
\hline Chronic renal disease & 40 & (3.2) \\
\hline Pregnancy $(\mathrm{N}=669)^{*}$ & 80 & $(12.0)$ \\
\hline \multicolumn{3}{|l|}{ Number of medical conditions } \\
\hline None & 550 & $(44.5)$ \\
\hline One & 524 & (42.4) \\
\hline Two & 117 & (9.5) \\
\hline Three & 37 & (3.0) \\
\hline Four & 8 & $(0.6)$ \\
\hline \multicolumn{3}{|l|}{ Antiviral treatment $(\mathrm{N}=842)$} \\
\hline Yes & 737 & $(87.5)$ \\
\hline No & 105 & $(12.5)$ \\
\hline \multicolumn{3}{|c|}{$\begin{array}{l}\text { Antiviral treatment initiated within } 48 \text { hours of onset } \\
(\mathrm{N}=842)\end{array}$} \\
\hline Yes & 561 & $(66.7)$ \\
\hline No & 281 & (33.3) \\
\hline
\end{tabular}

disease onset compared to those that were not admitted to ICU or SCU (Table 2). The median time from the onset of illness to the initiation of antiviral therapy was 3 days (range, zero to 28 days) for patients admitted to ICU or SCU. Among patients who were admitted to ICU or SCU, $12 \%(67 / 189)$ received antiviral drugs within 48 hours of the onset of illness. There was no
Table2. Characteristics and univariate analysis of factors associated with ICU/SCU admission in hospitalized patients with confirmed pandemic A(H1N1) 2009 infection, Queensland, Australia (May to October 2009)

\begin{tabular}{|c|c|c|c|c|}
\hline & \multicolumn{4}{|c|}{ Admission to ICU or SCU } \\
\hline & No & Yes & Yes (\%) & OR $(95 \% \mathrm{Cl})^{*}$ \\
\hline \multicolumn{5}{|l|}{ Hospital type } \\
\hline Public & 857 & 162 & 14.6 & $1.9(0.6-5.6)$ \\
\hline Private & 190 & 27 & 16.5 & 1 \\
\hline \multicolumn{5}{|l|}{ Age (years) } \\
\hline Less than 10 & 296 & 7 & 2.3 & $0.52(0.16-1.7)$ \\
\hline $10-17$ & 110 & 5 & 4.3 & 1 \\
\hline $18-44$ & 308 & 75 & 19.6 & $5.4(2.1-13.5)$ \\
\hline $45-64$ & 233 & 87 & 27.2 & $8.2(3.2-20.8)$ \\
\hline $65+$ & 69 & 24 & 25.8 & $7.7(2.8-21.0)$ \\
\hline \multicolumn{5}{|l|}{ Sex } \\
\hline Male & 471 & 91 & 16.2 & 1 \\
\hline Female & 548 & 110 & 16.7 & $1.3(0.8-1.9)$ \\
\hline \multicolumn{5}{|c|}{ Indigenous status } \\
\hline Indigenous & 165 & 26 & 15.2 & $1.2(0.5-1.4)$ \\
\hline Non-indigenous & 784 & 140 & 16.4 & 1 \\
\hline \multicolumn{5}{|c|}{ Chronic respiratory disease } \\
\hline Yes & 335 & 60 & 14.5 & $1.4(0.4-1.1)$ \\
\hline No & 578 & 115 & 17.7 & 1 \\
\hline \multicolumn{5}{|l|}{ Diabetes } \\
\hline Yes & 96 & 26 & 21.3 & $1.2(0.7-2.2)$ \\
\hline No & 815 & 143 & 14.9 & 1 \\
\hline \multicolumn{5}{|l|}{ Pregnant $^{\dagger}$} \\
\hline Yes & 61 & 17 & 21.3 & $1.1(0.4-2.6)$ \\
\hline No & 442 & 85 & 12.7 & 1 \\
\hline \multicolumn{5}{|c|}{ Immunocompromised } \\
\hline Yes & 79 & 8 & 9.2 & $1.4(0.3-1.3)$ \\
\hline No & 834 & 161 & 16.2 & 1 \\
\hline \multicolumn{5}{|l|}{ Morbid obesity } \\
\hline Yes & 37 & 23 & 38.3 & $3.6(2.1-6.2)$ \\
\hline No & 871 & 151 & 14.8 & 1 \\
\hline \multicolumn{5}{|c|}{ Chronic renal disease } \\
\hline Yes & 27 & 13 & 32.5 & $3.7(1.8-6.8)$ \\
\hline No & 882 & 156 & 15.0 & 1 \\
\hline \multicolumn{5}{|c|}{ Chronic cardiac disease } \\
\hline Yes & 73 & 24 & 30.2 & $2.4(1.5-3.8)$ \\
\hline No & 840 & 144 & 15.3 & 1 \\
\hline \multicolumn{5}{|c|}{ Antiviral treatment initiated within 48 hours of onset } \\
\hline Yes & 484 & 69 & 12.5 & 1 \\
\hline No & 194 & 85 & 30.5 & $2.1(1.4-3.0)$ \\
\hline
\end{tabular}

*Odds Ratio and 95\% Confidence Interval; ${ }^{\dagger}$ Only female Note: numbers may not add to total due to missing data

significant difference in the proportion of indigenous and non-indigenous patients admitted to ICU or SCU.

Forty-one patients (3\%) hospitalized with pandemic (H1N1) 2009 infection died. The median age of patients who died was 51 years (range 13 to 84 years); the median time from the onset of illness to death was 
Table 3. Multivariate analysis of factors associated with admission to ICU or SCU in hospitalized patients with confirmed pandemic A(H1N1) 2009 infection, Queensland, Australia (May to October 2009)

\begin{tabular}{lc}
\hline & OR $(95 \% \mathrm{Cl})^{*}$ \\
\hline Age (years) & $0.1(0.1-0.7)$ \\
Less than 10 & 1 \\
$10-17$ & $3.9(1.4-11.2)$ \\
$18-44$ & $7.1(2.6-21.3)$ \\
$45-64$ & $5.7(1.8-18.2)$ \\
$65+$ & 1 \\
\hline Antiviral treatment initiated within 48 hours of onset \\
Yes $^{\dagger}$ & $2.1(1.34-3.3)$ \\
No & 1 \\
\hline Chronic renal disease & 1 \\
No $^{\dagger}$ & $2.7(1.3-5.7)$ \\
Yes $^{+}$ & 1 \\
\hline Morbid obesity & $1.9(1.4-3.6)$ \\
\hline No & \\
Yes &
\end{tabular}

* Odds Ratio and 95\% Confidence Interval; ${ }^{\dagger}$ Reference group

14 days (range 1 to 68 days) and 27 (67\%) had an underlying medical condition. Of the patients who died, $80 \%$ had received antiviral drugs. Among the patients who died who received antiviral treatment, the median time from the onset of illness to the initiation of antiviral therapy was 3 days (range, less than 1 day to 28 days). Forty-eight per cent of those who died received antiviral therapy within 48 hours of the onset of symptoms.

Patients with any severe outcome (defined as those admitted to an ICU or SCU plus those who died) were older (median age 50 years, range from less than 1 to 85 ) and had a longer median time (4 days, range between less than 1 day to 28 days) between onset of illness and the initiation of antiviral therapy, compared to other hospitalized patients, in whom the median age was 30 years (range from less than 1 to 90) and the median time between onset and receiving antiviral drugs was 1 day (range from less than 1 day to 33 days).

Tables 2 and $\mathbf{3}$ show the univariate and multivariate results of the logistic regression analysis. In the unadjusted model, factors significantly associated with increased likelihood of admission to ICU or SCU were older age, chronic renal disease, chronic cardiac disease, morbid obesity and not receiving antiviral treatment
Figure 1. Receiver-operating characteristic curve for multivariate logistic regression model to predict admission to ICU or SCU in hospitalised patients with confirmed pandemic $A(H 1 N 1) 2009$ infection, Queensland, Australia (May to October 2009) [Area under curve $=0.76(95 \%$ Confidence Interval $=0.72-0.79 ; P<0.001)]$

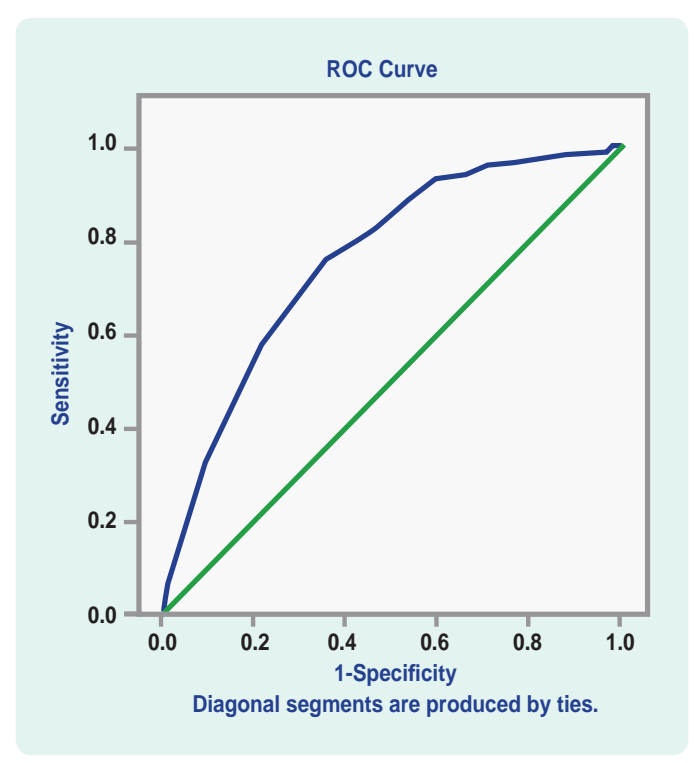

within 48 hours of onset. When these variables were included in a single model adjusting for each other, all factors remained significant except for chronic cardiac disease. Compared to patients who had received antiviral treatment within 48 hours of onset, patients who had not received antiviral drugs or received antiviral treatment later than 48 hours of onset were twice as likely to be admitted to ICU or SCU. A ROC curve was developed (Figure 1) from the fitted predicted value from the final logistic regression model. The area under the operator characteristic curve was 0.76 .

\section{DISCUSSION}

Using data from a newly established hospitalisation surveillance system, we examined the epidemiological profile of 1236 patients admitted to hospitals in Queensland, Australia with confirmed pandemic $\mathrm{A}(\mathrm{H} 1 \mathrm{~N} 1) 2009$ infection. We found that $34 \%$ of the hospitalized patients were under the age of 18 years, more than one third were between the ages of 18 and 49 years, and only $8 \%$ were 65 years of age or older. This pattern is similar to results reported elsewhere. ${ }^{4-6}$

Pregnancy has been associated with increased hospitalization and severity of illness due to pandemic 
$\mathrm{A}(\mathrm{H} 1 \mathrm{~N} 1) 2009$ infection. ${ }^{4,9-11}$ In our study, $7 \%$ of hospitalized patients with confirmed pandemic $A(H 1 N 1) 2009$ infection were pregnant compared to the $1 \%$ prevalence of pregnancy in the general population. ${ }^{12}$ Although a slightly higher proportion of pregnant patients were admitted to ICU or SCU, this did not reach statistical significance.

We found that morbid obesity and chronic renal disease were associated with increased odds of admission to ICU or SCU. Morbid obesity has been associated with increased severity of illness due to pandemic A(H1N1) 2009 infection in previous studies., 4,5,13-15 Similarly, chronic renal disease has also been reported as a potential risk factor for severity of illness due to pandemic $A(\mathrm{H} 1 \mathrm{~N} 1) 2009$ infection. ${ }^{15,16}$

Considerably higher rates of hospital admission due to pandemic A(H1N1) 2009 influenza infection have been reported among indigenous people in other Australian states, ${ }^{15,16}$ New Zealand and Canada compared to those of European descent. ${ }^{14,17}$ This is possibly due to a higher prevalence of medical risk factors, poorer access to primary health care services and socioeconomic factors in this community. ${ }^{18}$ However, we found that the proportion of hospitalized patients admitted to ICU or SCU was similar among indigenous and non-indigenous Queenslanders. Missing data from indigenous status may influence the proportion of hospital admission recorded as indigenous. Ten per cent of data on indigenous status was unknown or missing which limited our interpretation of the association between ICU/SCU admission and indigenous status.

Our final logistic regression model revealed that factors such as older age, initiation of antiviral treatment later than 48 hours and presence of morbid obesity and/ or chronic renal disease were significantly associated with admission to ICU/SCU. This model had reasonable predictive accuracy for admission to ICU or SCU, with the area under the ROC curve being 0.76 ( 1 indicating perfect predictive accuracy and 0.5 no predictive accuracy above chance). ${ }^{19}$

Our analysis has several limitations. Analysis was undertaken using only data on patients with laboratoryconfirmed pandemic A(H1N1) 2009 infection. This may underestimate the true number of cases as some may not have been tested or have tested negative due to technical or timing factors. The data on underlying medical conditions were from patient self-report and were not clinically validated. Body mass index values were not recorded, so it is unclear what role clinical judgement may have played in categorization of morbid obesity (or other medical conditions for that matter). There is also the possibility of reporting bias, which may results in confounding in our logistic regression model. Despite the use of a standardized data collection template, not all information was captured for all cases and some data fields were incomplete. The study used data from two sources: EpiLog for public hospitals and separately submitted spreadsheets for private hospitals. It was not possible to verify the data recorded. Finally, as socialdemographic data were collated by linking the EpiLog system and HBCIS missing data were unavoidable.

Despite these limitations, EpiLog provides an example of a new surveillance application that was rapidly developed to meet an urgent public health need. It enabled close to real-time monitoring of the severity of pandemic A(H1N1) 2009 infection and its impact on Queensland's hospital system and critical care services. It was possible to monitor the progress of the pandemic and gain rapid information on risk factors for severe disease and patient outcomes. Reflection on the use of EpiLog during the 2009 influenza pandemic led to several enhancements of the application. Many of the improvements related to streamlining the interface between EpiLog and HBCIS and the efficient management of data quality and reporting issues.

Finally, there is growing evidence that early treatment with antiviral medication may reduce the likelihood of hospitalization and death due to pandemic $A(H 1 N 1) 2009$ infection. ${ }^{5,20}$ Our study supports these findings. Patients admitted to an ICU or SCU, or who died, were less likely to have received antiviral therapy within 48 hours of the onset of symptoms compared to other hospitalized patients. Early antiviral treatment for influenza cases may therefore have important public health implications for example for reducing the severity of the impact of pandemic $A(H 1 N 1) 2009$ infection.

\section{Conflicts of interest}

None declared.

\section{Funding}

None. 


\section{References:}

1. Appuhamy RD et al. The changing phases of pandemic (H1N1) 2009 in Queensland: an overview of public health actions and epidemiology. The Medical Journal of Australia, 2010, 192: 94-97. doi:10.1126/science.3277268 pmid:20078411

2. Cao B et al.; National Influenza A Pandemic (H1N1) 2009 Clinical Investigation Group of China. Clinical features of the initial cases of 2009 pandemic influenza $A(H 1 N 1)$ virus infection in China. New England Journal of Medicine, 2009, 361:2507-2517. doi:10.1056/NEJMoa0906612 pmid:20007555

3. Louie JK et al.; California Pandemic (H1N1) Working Group. Factors associated with death or hospitalization due to pandemic 2009 influenza $A(\mathrm{H} 1 \mathrm{~N} 1)$ infection in California. JAMA: The Journal of the American Medical Association, 2009, 302:18961902. doi:10.1001/jama.2009.1583 pmid:19887665

4. Fuhrman $C$ et al. Severe hospitalised 2009 pandemic influenza A (H1N1) cases in France, 1 July-15 November 2009. Euro Surveillance: European Communicable Disease Bulletin, 2010, 15(2):17-21. pmid:20085690

5. Zarychanski $\mathrm{R}$ et al. Correlates of severe disease in patients with 2009 pandemic influenza (H1N1) virus infection. Canadian Medical Association Journal, 2010, 182:257-264. doi:10.1503/ cmaj.091884 pmid:20093297

6. Campbell A et al. Risk of severe outcomes among patients admitted to hospital with pandemic (H1N1) influenza. Canadian Medical Association Journal, 2010, 182:349-355. doi:10.1503/ cmaj.091823 pmid:20159893

7. Hanley JA, McNeil BJ. The meaning and use of the area under a receiving operating characteristic (ROC) curve. Radiology, 1986, 50:23-36.

8. Statistical Package for Social Scientist, Chicago.

9. Jamieson DJ et al.; Novel Influenza A (H1N1) Pregnancy Working Group. H1N1 2009 influenza virus infection during pregnancy in the USA. Lancet, 2009, 374:451-458. doi:10.1016/S01406736(09)61304-0 pmid:19643469

10. Webb SA et al; ANZIC Influenza Investigators. Critical care services and 2009 H1N1 influenza in Australia and New Zealand. The New England Journal of Medicine, 2009, 361:1925-1934. doi:10.1056/NEJMoa0908481 pmid:19815860

11. Kumar A et al. Critically ill patients with 2009 influenza A (H1N1) infection in Canada. JAMA: The Journal of American Association,
2009, 302: 1872-1879. doi:10.1001/jama.2009.1496 pmid:19822627

12. Australian Bureau of Statistics. 3201.0 - population by age and sex, Australian states and territories, June 2008. (http:// www.abs.gov.au/AUSSTATS/abs@.nsf/MF/3201.0, accessed on 21 March 2011).

13. Centers for Disease Control and Prevention. Intensive-care patients with severe novel influenza $A(\mathrm{H} 1 \mathrm{~N} 1)$ virus infection Michigan June 2009. MMWR. Morbidity and Mortality Weekly Report, 2009, 58:749-752.

14. Morgan $\mathrm{OW}$ et al. Morbid obesity as a risk factor for hospitalization and death due to 2009 pandemic influenza $A(\mathrm{H} 1 \mathrm{~N} 1)$ disease. PLOS ONE, 2010, 5:9694. doi:10.1371/journal.pone.0009694 pmid:20300571

15. Australian Government Department of Health and Ageing. Australian influenza surveillance summary report, no. 21, 2009, reporting period: 26 September 2009-2 October 2009. (http://www.healthemergency.gov.au/internet/health emergency/publishing.nsf/Content/18D06BAC4644C98DCA 25763E00823442/\$File/ozflu-no21-2009.pdf, accessed on 21 March 2011).

16. Flint SM et al. Disproportionate impact of pandemic (H1N1) 2009, influenza on Indigenous people in the Top End of Australia's Northern territory. Medical Journal of Australia, 2010. (http:// www.mja.com.au/public/issues/192_10_170510/fli10103_ fm.html, accessed 21 March 2011).

17. Baker MG et al. Pandemic influenza A(H1N1)v in New Zealand: the experience from April to August 2009. Euro Surveillance: European Communicable Disease Bulletin, 2009; 14(34): pii $=19319$. pmid: 19712648

18. Australia's health 2008. Cat.no. AUS 99. Canberra: Australian Institute of Health and Welfare, 2008.

19. Harrell FE Jr, Lee KL, Mark DB. Multivariable prognostic models: issues in developing models, evaluating assumptions and adequacy, and measuring and reducing errors. Statistics in Medicine, 1996, 15:361-387. doi:10.1002/(SICI)10970258(19960229) 15:4<361::AID-SIM168>3.0.CO;2-4 pmid:8668867

20. Jain S et al.; 2009 Pandemic Influenza A (H1N1) Virus Hospitalizations Investigation Team. Hospitalized patients with 2009 H1N1 influenza in the United States, April-June 2009. The New England Journal of Medicine, 2009, 361:1935-1944. doi:10.1056/NEJMoa0906695 pmid:19815859 